\title{
LIQUID METAL TARGET FOR NLC POSITRON SOURCE
}

\author{
V. Belov, V. Karasyuk, V. Kobets, G. Kraynov, P. Martishkin, M. Petrichenkov, G. Silvestrov, \\ T. Sokolova, T. Vsevolozhskaya, G. Willewald \\ BINP, Novosibirsk, Russia,
}

A. Bochko, IMET RAS ,Moscow,Russia

J. Sheppard, V. Bharadwaj, D. Schultz, SLAC,USA

\begin{abstract}
Possibility of creating the liquid lead target with parameters, optimum for the NLC positron source, is investigated. Target has a form of titanium vessel, filled with liquid lead, pumped through. The energy deposition in target is characterized by $35 \mathrm{~kW}$ average power and up to $250 \mathrm{~J} / \mathrm{g}$ specific energy at optimum beam sigma $0.6 \mathrm{~mm}$. The use of pumped through liquid lead as target material solves both the problems of power evacuation and target survival. The window for beam exit is made of both temperature and pressure resistive material - the diamond-like ceramic $\mathrm{BN}$.
\end{abstract}

The optimal conditions for positron capture in a frame of the concept of new NLC positron source [1] include the small size of primary electron beam $\left(\sigma_{\mathrm{b}}=0.6 \mathrm{~mm}\right)$ and small, practically null, distance between the target output and the maximum of magnetic field in flux concentrator. The last means that the end of target has to be situated inside the concentrator. This confines the outer transverse size of target by concentrator aperture, i.e._ less than $10 \mathrm{~mm}$. For $1.33 \cdot 10^{12}$ particles in electron beam at $6.2 \mathrm{GeV}$ energy the specific energy deposition achieves $\sim 250 \mathrm{~J} / \mathrm{g}$. Such an energy, released during a short $-260 \mathrm{~ns}-$ time of particle pulse besides the high temperature rise results in high several tens kbar - pressure, which no solid target material can survive. Average released power is equal to $\sim 35 \mathrm{~kW}$.

The only possibility to provide simultaneous solution of mechanical and thermal problems of target consists in the use of liquid lead target with the lead pumped through. Here, however, with the target material destroy being eliminated, the problem of target envelope survival arises, especially for the window at output target end, which separates the liquid lead volume from high-vacuum region of linear accelerator. Besides that the problem of window cooling is to be solved, which gets complicated by small size of the device.

The design option of liquid lead target, which solves these problems, is presented in Fig.1. The target volume, excluding the part, adjacent to the window, is divided into two coaxial parts with the use of thin-wall titanium cylinder. The "cold" lead is pumped into the outer part, while the "hot" is removed through the inner one. Over the window surface the lead is flowing along the radius in direction to the center. The absolute value of radial velocity, equal to zero at the axis, grows about linearly with radius at small $\mathrm{r}$, achieving its maximum value

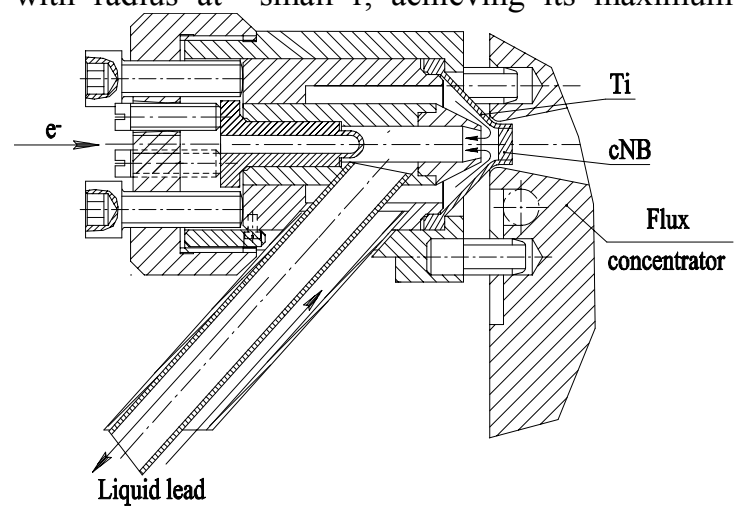

Fig.1. Cross section of liquid lead target.

near $r$ equal to radius of separating cylinder. When the cross sections of both the outer and inner coaxial parts are equal to each other and to the cross section of connecting strait, the maximum absolute value of $u_{\mathrm{r}}$ is equal to about half of the initial longitudinal velocity $u_{0}$ of lead flow. The lower limit of $u_{0}$ is defined by the necessity to evacuate the heated lead from operational target volume between the particle pulses. With target length equal to $\sim 30 \mathrm{~mm}$ and $120 \mathrm{~Hz}$ repetition rate this lower limit is $\sim 4 \mathrm{~m} / \mathrm{s}$.

Zero magnitude of lead velocity near the beam axis, where the energy density is maximum, gives rise to a worry about the efficiency of cooling of window. However, the radial flow of chosen direction leads to a rapid decrease with time of transverse size of heated region $\sigma_{\text {eff }}$ from the initial value $\sigma_{0}$ to the final one $a^{2} / \mu$ as follows:

$$
\sigma_{e f f}^{2}=\sigma_{0}^{2} \cdot\left[\left(1-e^{-2 \mu t}\right) \frac{a^{2}}{\mu \sigma^{2}}+e^{-2 \mu t}\right]
$$

Here $a^{2}$ denotes the temperature conductivity, and $\mu$ stands for the absolute value of radial velocity derivative, $\mu=-\partial u_{\mathrm{r}} / \partial r$. In geometry under consideration $\mu=2.4 \mathrm{~ms}^{-1}$ when velocity of lead flow is equal to $10 \mathrm{~m} / \mathrm{s}$,. The final 
value of $\sigma_{\text {eff }}$, by that, is less than $0.1 \mathrm{~mm}$. This provides very high temperature gradient and very efficient cooling of lead through the thermal conductivity. In result, the temperature at $r=0$,

$$
T(0, t)=\frac{\sigma^{2}}{\sigma_{e f f}^{2}} T(0,0) \cdot \exp \left(-2 \mu t-\frac{r^{2}}{2 \sigma_{e f f}^{2}}\right)
$$

retains high value after the energy release only for a rather short time period $\sim 1 \mathrm{~ms}$.

The longitudinal velocity in lead is also linearly growing (with coordinate $z$ ) near the window surface. Its derivative is equal to $2 \mu, \partial u_{z} / \partial z \cong 2 \mu$. With an account made for both - the radial and axial - matter velocities, the equation for thermal conductivity in the lead volume, adjacent to the central part of window, gets a form:

$$
\frac{\partial T}{\partial t}=a^{2} \Delta T+\mu r \frac{\partial T}{\partial r}-2 \mu z \frac{\partial T}{\partial z},
$$

whose solution is expressed through the singular hypergeometric functions. Symbol $\Delta$ here stands for the Laplacian unlike the symbol $\Delta$, used below for temperature rise.

The temperature rise $\Delta T$ in the lead directly after the particle pulse of short duration is defined by the whole deposed specific energy and is equal to $\sim 1940^{\circ} \mathrm{C}$ at the beam axis, when $Q_{0} \cong 250 \mathrm{~J} / \mathrm{g}$. Then, within a time of the order of $\sigma / c_{0}$, i.e. less than $1 \mu \mathrm{s}$, while the matter expands until the pressure becomes equal to zero, the $\Delta T$ gets adiabatic decrease down to a value $\Delta T_{\mathrm{t}} \sim 1350^{\circ} \mathrm{C}$ at the beam axis. This is the initial point for cooling through thermal conductivity and liquid lead flow. With initial temperature of liquid lead equal to $\sim 350^{\circ} \mathrm{C}$ (or $250^{\circ} \mathrm{C}$ for lead alloy), the final temperature in lead appears to be $\sim 1700^{\circ} \mathrm{C}\left(\sim 1600^{\circ} \mathrm{C}\right.$ for lead alloy).

The temperature rise at the common surface of target and window is defined through both their own temperature rises (see below), and appears to be sufficiently dependent on relation between the characteristics of target and window matters.

The window has to be made of substance with low density, high tensile strength and good thermal conductivity. The diamond-like cubic boron-nitride was chosen as a material for window. Its characteristics are: $\gamma=3.5 \mathrm{~g} / \mathrm{cm}^{3}$, thermal conductivity $\lambda=300 \mathrm{~W} / \mathrm{m} \cdot \mathrm{deg}$, melting point $T_{m}=3000^{\circ} \mathrm{C}$, thermal expansion coefficient $\alpha=1.8 \cdot 10^{-6}{ }^{\circ} \mathrm{C}^{-1}$. Tensile strength is $40 \mathrm{kbar}$ at normal temperature and $\sim 10 \mathrm{kbar}$ at $2000^{\circ} \mathrm{C}$.

The energy deposition in the window matter itself directly near the surface, adjacent to target, is determined by a shower, extended within the lead. So, its specific value $Q$ differs from that in the lead by a ratio of mean rates of ionization losses in both matters, that is by $\sim 1.3$ times. The shower in the lead to the most number of particles consists of very low energy ones, which are stopped in window matter within a very short distance $\sim 0.1 \mathrm{~g} / \mathrm{cm}^{2}$, that is $\sim 0.3 \mathrm{~mm}$ in cubic boron-nitride. Thus the deposed energy density reduces rapidly with distance from target surface down to a level of $\sim 0.2$ [1]. The temperature rise in flange matter, caused by the energy deposition in it, is equal to $\Delta T_{\mathrm{w}} \sim 690^{\circ} \mathrm{C}$ near the inner surface and $\sim 140^{\circ} \mathrm{C}-$ near the outer. Power, released in window matter, is equal to $\sim 0.7 \mathrm{~kW}$.

The solution of two-dimensional boundary-value problem for thermal conductivity cannot be obtained in analytic form. The use of operator method of complex variable function theory permits to obtain the integral equation $\quad \int_{0}^{t} T_{q}(z, \tau) W(t-\tau) d \tau=F_{q}(z, t) \quad$ for the component $T_{q}$ of integral (or in series) development of temperature with respect to the Bessel functions, $T_{q}(z, t)=\int_{0}^{\infty} T(r, z, t) J_{0}(q r) r d r$ The equation solution as well as integration over $q$ are obtained numerically.

The temperature rise at the common surface of target and window just after the particle pulse is found equal to:

$$
\Delta T_{\text {surf }}=\frac{\Delta T_{w} \cdot\left(\sqrt{\lambda \gamma c_{v}}\right)_{w}+\Delta T_{t} \cdot\left(\sqrt{\lambda \gamma c_{v}}\right)_{t}}{\left(\sqrt{\lambda \gamma c_{v}}\right)_{w}+\left(\sqrt{\lambda \gamma c_{v}}\right)_{t}},
$$

expressed through their own temperature rises $T_{\mathrm{w}}$ and $T_{\mathrm{t}}$.

Temperature at the beam axis for several values of longitudinal coordinate $z$ in the window is shown versus time in Fig.2. The lead flow velocity is equal to $10 \mathrm{~m} / \mathrm{s}$.

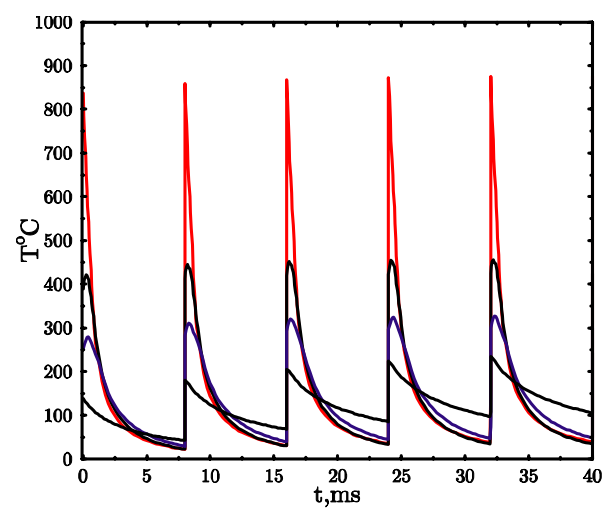

Fiq.2. Temperature rise versus time by the window axis at $z$ equal to 0 (inner surface), $0.25 \mathrm{~mm}, 0.5 \mathrm{~mm}$ and $4 \mathrm{~mm}$ (outer surface) by initial velocity $u_{0}$ of lead flux equal to $10 \mathrm{~m} / \mathrm{s}$.

It is seen, that the cooling is quite efficient. The maximum steady state rise of temperature level - by outer window surface - does not exceed $150^{\circ} \mathrm{C}$ at $120 \mathrm{~Hz}$ repetition rate. Besides this, the high temperature - of the order of $1250^{\circ} \mathrm{C}$ - takes place within a very thin layer of window matter, adjacent to the lead surface. This is rather fortunate, because though the tensile strength here is reduced, the tension is also very low near the surface of liquid lead (see below), wherein the pressure is never negative.

The pressure, arising in target after the particle pulse of infinitely short duration, $\tau<<\sigma / c_{0}(\sigma$ is the r.m.s. transverse size of energy deposition area and $c_{0}$ - the sound velocity), is defined by the deposed specific energy $Q_{0}$, as: $P_{0}=Q_{0} \cdot \Gamma / V$ [2]. Here $\Gamma$ stands for the Gruneisen coefficient and $V$ - for the specific volume $(V=1 / \gamma)$ of target matter. With $Q_{0} \cong 250 \mathrm{~J} / \mathrm{g}$ in lead the value of $P_{0} \cong$ 
$70 \mathrm{kbar}$. By finite pulse duration the maximum pressure is less than $P_{0}$ by a factor, dependent on $\tau$ in $\sigma / c_{0}$ units. For $\tau \cong 260 \mathrm{~ns}$ and $\sigma \cong 0.9 \mathrm{~mm}$ in lead this factor is $\sim 0.9$, and maximum pressure is equal to $\sim 63 \mathrm{kbar}$. The pressure falls down to zero in a time of the order of $\sigma / c_{0}$ after the pulse finish. In solid targets it continues falling below zero, which means the tension arising. Near the free target end, where the reflected wave of decompression adds essentially to the negative pressure, its value achieves $P_{\min } \cong-1.28 P_{0}$ in the case of infinitely short pulse. This value, when compared with the limit tensile strength of material, defines the ultimate permissible specific energy deposition in solid target by short spill duration. For tungsten-rhenium alloy [3], for instance, this magnitude is less than $50 \mathrm{~J} / \mathrm{g}$. For the case under consideration $(\tau \sim 260 \mathrm{~ns}, \sigma \sim 0.9 \mathrm{~mm})$ the absolute value of maximum negative pressure is by $30 \%$ less than the above value $P_{\min }$. This makes the acceptable specific energy deposition higher up to $\sim 70 \mathrm{~J} / \mathrm{g}$, which is still very far from the optimum value.

For liquid target the problem of its destruction is eliminated, but the problem of envelope, especially, the window survival, is to be solved. When the window is made of rather light material - such as diamond-like ceramics - the pressure in its matter is mainly caused by the compressive wave, penetrating from target body through the common surface. Here also the solution of two-dimensional boundary-value problem of pressure wave propagation is obtained in a form of integral equation for $q$-component of pressure, which is computed numerically. Directly at the inner surface of window the pressure is close to $\sim 0.5 P_{0}$, reducing with distance the quicker the more is the particle pulse duration in $\sigma / c_{0}$ units. Simultaneously reduces the negative pressure in decompressive wave, reflected from free outer window surface.

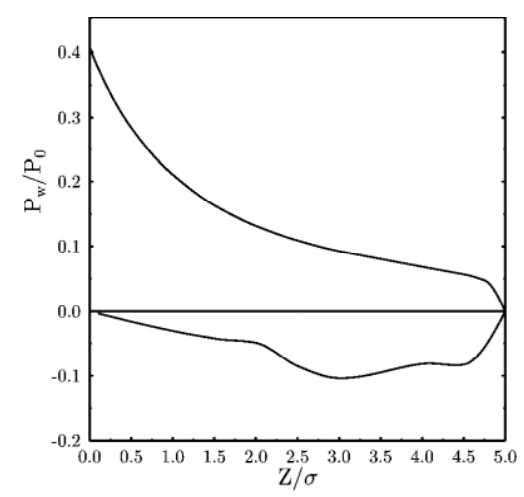

Fig.3 Maximum and minimum pressure at the window axis versus the longitudinal coordinate in $\sigma$ units.

The maximum and minimum pressure at the window axis are presented in Fig. 3 versus $z$ in $\sigma$ for 260 ns particle pulse duration and $0.9 \mathrm{~mm} \sigma$ of energy deposition region (the electron beam sigma $\sigma_{b} \cong 0.6 \mathrm{~mm}$ ), and $4.5 \mathrm{~mm}$ $(\sim 5 \sigma)$ window thickness. The negative pressure magnitude in flange of diamond-like ceramics does not exceed $\sim 0.1 P_{0}$. With $Q_{0} \cong 250 \mathrm{~J} / \mathrm{g}$ this means
$-P_{\min } \cong-7 \mathrm{kbar}$, which is well lower than tensile strength of cubic boron-nitride at temperature $\sim 2000^{\circ} \mathrm{C}$, whenever the real temperature in a region of maximum amplitude of negative pressure and, thus, maximum tension does not exceed $600^{\circ} \mathrm{C}$.

The target device (Fig. 4) includes three targets, operating independently and being changed automatically in case of output window destruction. Each target is supplied by liquid lead, but lead is pumped only through one of them which is placed in operational position and heated. The heaters of reserve targets are turned off normally and switched on only in case of targets replacement.

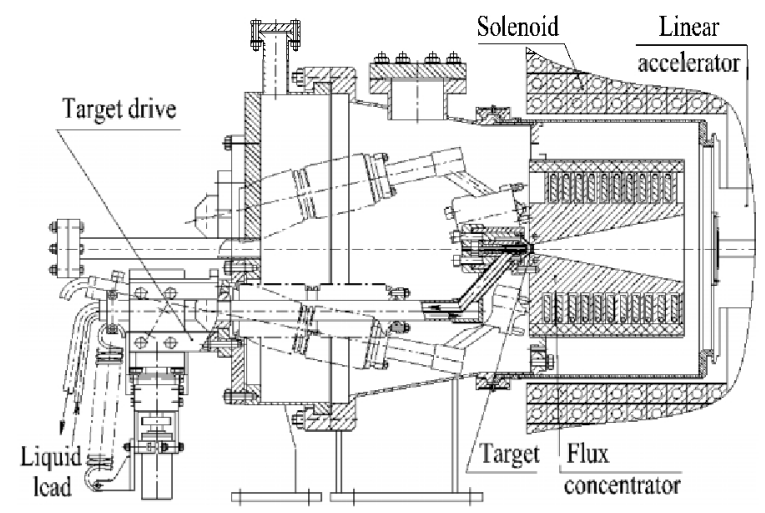

Fig. 4 Liquid lead target device

The serious problem for target under consideration is the survival of separating cylinder and the outer envelope of target. By small transverse size of target the cylinder radius is only by 2-3 times larger than $\sigma$ of energy deposition region, so the wave, reaching the cylinder, retains sufficiently high pressure $\sim 15 \mathrm{kbar}$. Under its action the cylinder radius gets an increase of the order of $0.1 \mathrm{~mm}$, i.e. $\sim 4 \%$, and the same is the linear deformation of cylinder surface. The tension, caused by such deformation exceeds the permissible value for titanium. To avoid the cylinder destruction it may be multiply cut longitudinally to provide higher radial elasticity.

The outer envelope in its narrow part also gets a tension well above the permissible magnitude for titanium. The best solution here seems to be the fabrication of cubic boron-nitride not only the window but the whole small radius head of target.

\section{REFERENCES}

[1] V. Belov, V. Eschenko, V. Karasyuk, et al. Concept of New NLC Positron Source. HEACC 2001, Tsukuba, Japan, March, 2001.

[2] Ya. B. Zeldovitch and Yu. P. Rizer, Physics of Shock Waves and High Temperature Phenomena, Science, Moscow, 1966

[3] E.M. Savitsky, K.V. Povarova, P.V. Makarov, Metallurgy of Tungsten, Moscow, "Metallurgy", 1978 